\title{
Conocimientos que tienen médicos veterinarios no vinculados a la salud pública en la provincia Ciego de Ávila, Cuba sobre algunas zoonosis.
}

\section{Artículo Original}

Miguel Suárez-Hernández ${ }^{1}$, Francisco Llorens-Blanco², Omelio Cepero-Rodríguez ${ }^{2}$, Marta ReturetaMilian $^{3}$, Tayry González-Marti ${ }^{4}$

${ }^{1}$ Centro Provincial de Higiene y Epidemiología de Ciego de Ávila, ${ }^{2}$ Universidad Central de las Villas, ${ }^{3}$ Centro Provincial de Higiene y Epidemiología, ${ }^{4}$ Dirección Nacional de Epidemiología. Cuba.

\section{RESUMEN.}

La presente investigación es un diseño descriptivo y se realizó en la provincia de Ciego de Ávila para evaluar el nivel de conocimientos sobre las zoonosis de importancia médica en la provincia, en médicos veterinarios no vinculados al sector salud. El universo fueron 211 veterinarios, y la muestra incluyó a 107 de ellos. A cada seleccionado se le aplicó un cuestionario que había sido previamente validado. El instrumento de medición tenía 150 preguntas y se le dio un valor de 1 punto a cada inciso. Se consideró aprobado el que tuviera $70 \%$ o más de los puntos, y también se midió la aprobación por las diferentes entidades patológicas. Se relacionó la aprobación del cuestionario con el tiempo desde la graduación.

El $31 \%$ de los estudiados aprobaron el cuestionario; la proporción de aprobados en el grupo de más de 15 años desde su graduación fue superior, sin llegar al umbral de significación estadística.

De las zoonosis evaluadas la mejor información fue sobre fasciolasis y criptosporidiosis y la menor en toxoplasmosis y rabia. Existió diferencia estadística en la aprobación de los diferentes temas.

(Rev Biomed 2005; 16:221-226)

Palabras clave: Médicos veterinarios, zoonosis, salud pública, Cuba.

\section{SUMMARY.}

Knowledge veterinarians unrelated to public health activities in the providence of Ciego de Avila, Cuba, have regarding some zoonoses.

This is a descriptive investigative design carried out in the province of Ciego de Avila, Cuba, to assess the knowledge veterinarians not directly

Solicitud de sobretiros: Dr. Miguel Suárez-Hernández. Serafín Sánchez 155 entre Onelio Hernández y Martí, Ciego de Ávila 2, 65200 Cuba. E-mail: epi.hig@trocha.cav.sld.cu

Recibido el 23/Mayo/2005. Aceptado para publicación el 13/Octubre/2005.

Este artículo está disponible en http://www.uady.mx/sitios/biomedic/revbiomed/pdf/rb051641.pdf

Vol.16/No.4/Octubre-Diciembre, 2005 


\section{Suárez-Hernández, F Llorens-Blanco, O Cepero-Rodríguez, M Retureta-Milian y col.}

involved in official human health practice have concerning the most important zoonoses in the province. 107 doctors were chosen from a universe of 211 and each one responded to a previously validated 150 item questionnaire.

Those who answered correctly $70 \%$ of the 150 questions or more were considered approved. The evaluation was also carried out regarding specific medical entities, and the association between knowledge and the date of graduation was also assessed.

$31 \%$ of doctors passed the questionnaire successfully and most of them had had their degrees for more than 15 years, yet this result was not statistically significant.

Fasciolasis and cryptosporidiosis were the entities with the best proportion of achievements while the worst results were related to toxoplasmosis and rabies. There were statistically significant differences in relation to the knowledge concerning different entities. (Rev Biomed 2005; 16:221-226)

Key words: Veterinarians, zoonoses, public health, Cuba.

\section{INTRODUCCIÓN.}

En correspondencia con los cambios ocurridos en la salud publica de Cuba y para mantener y ampliar los logros alcanzados es necesario ocupar nuevos espacios mediante la incorporación de técnicas actuales y promover la interrelación con otras ciencias (1)

Las ciencias veterinarias son vitales para el control de los alimentos de origen animal y las enfermedades que se transmiten al hombre a través de los animales. Con el desarrollo de las ciencias médicas y veterinarias se ha incrementado el conocimiento científico sobre las zoonosis (2).

Las zoonosis y las enfermedades transmisibles comunes al hombre y a los animales continúan registrando altas tasas de incidencia y causando significativa morbilidad y mortalidad en la población, así como pérdidas considerables en la economía (3).
En Cuba y en la provincia las principales zoonosis de importancia médica son la rabia, leptospirosis, brucelosis, salmonelosis, toxoplasmosis, teniosis- cisticercosis, fasciolosis y criptosporidiosis (4). La situación epidemiológica de las entidades señaladas en la provincia es la siguiente: rabia- no hay presentación de casos humanos después de 1959. La enfermedad se presenta en mangostas (Herpestes auropunctatus). La rabia canina es esporádica. Leptospirosis: entidad con alta morbilidad en la década de los 80 y 90 que cuyo reporte decreció como consecuencia de un conjunto de medidas, entre las que se incluye la vacunación de las personas expuestas. Salmonelosis: la fiebre tifoidea presenta bajo reporte de casos, en zonas limitadas; no hay fiebre paratifoidea; las salmonelosis zoonóticas tienen alta frecuencia. Toxoplasmosis: las formas clínicas más frecuentemente detectadas son la forma ganglionar y las oftalmopatías. Teniosis- cisticercosis: el complejo ha mantenido una estabilidad. Está reportada la Taenia saginata, pero esta entidad, al igual que el Cisticercus bovis, tiene baja proporción de casos. Fasciolosis: enfermedad frecuente en bovinos y ovinos, no así en el humano donde la enfermedad es esporádica. No se corresponde la morbilidad animal con los casos humanos. Criptosporidiosis: enfermedad que se empezó a estudiar a partir de 1987. En la provincia se ha demostrado que es importante en niños menores de 1 año (5).

En la provincia de Ciego de Ávila se han realizado diferentes investigaciones sobre el nivel de información de la población de las zoonosis de importancia médica, así como estudios para evaluar los conocimientos que tienen los médicos sobre leptospira, rabia y otras zoonosis. Sin embargo, no se han monitoreado los conocimientos que tienen los médicos veterinarios que no trabajan en el sector salud sobre la salud pública veterinaria. El presente trabajo aborda esa temática.

\section{MATERIALES Y MÉTODOS.}

La provincia Ciego de Ávila de la Republica

\section{Revista Biomédica}




\section{Conocimientos de veterinarios sobre zoonosis.}

de Cuba tiene 10 municipios. El número de médicos veterinarios es 238 de los cuales trabajan en el sector Salud 27.

El universo de la presente investigación son los 211 médicos veterinarios que no laboran en salud pública. La muestra fueron 107 para un $50.7 \%$ del total.

A cada profesional objeto de la investigación se le aplicó un instrumento de medición donde se abordaron las siguientes zoonosis:

a) Rabia b) Leptospirosis c) Brucelosis d) Toxoplasmosis e) Teniasis- Cisticercosis

f) Fasciolasis g) Criptosporidiosis h) Salmonelosis

De las entidades señaladas se evaluaron los siguientes aspectos: Etiología, Epidemiología, Epizootiología, Medidas de Prevención y Control

Las 150 preguntas exploradas se desglosaron de la siguiente
a) Rabia 22 b)
b) Leptospirosis 27 c) Brucelosis

22 d) Toxoplasmosis 13 e) Teniasis- Cisticercosis

20 f) Fasciolasis 16 g) Criptosporidiosis 19 h) Salmonelosis 11

A cada pregunta se le dio un valor de 1 punto para un total de 150 puntos (100\%). Se consideró aprobado el que tuviera 105 puntos (70\%). Además se evalúo la aprobación por temas. Se consideró aprobado el que alcanzara el $70 \%$ o más de los puntos. Para conocer si existían diferencias entre las zoonosis exploradas se aplicó la prueba de $\mathrm{Z}$ entre dos entidades entre sí hasta abordar todas las combinaciones.

Se relaciona el total de preguntas por temas y las preguntas que fueron desaprobadas se consideró con tal condición cuando 76 ó menos médicos veterinarios la respondieron correctamente (menos del 70\% de los encuestados).

Se relacionó la aprobación del cuestionario con los años de graduados estableciéndose los siguientes rangos: a) 1 a 5 años, b) 6 a 10 años, c) 11 a 15 años, d) más de 15 años.

Se aplicó el método de diferencia de proporciones para evaluar si existían diferencias entre el tiempo de graduación y la aprobación del cuestionario.
Se agruparon los resultados en cuadros.

\section{RESULTADOS.}

En el cuadro 1 se describen las diferentes zoonosis, el total de preguntas y las que ocasionaron dificultades. El 50\% de las preguntas no fueron aprobadas por los encuestados. La mayor frecuencia de éstas la presentó la toxoplamosis, seguido de la rabia y leptospirosis.

La aprobación del cuestionario por temas es reflejada en la cuadro 2. El porcentaje de aprobados osciló desde un 33 hasta un $77 \%$. La mayor frecuencia de aprobados fue en fasciolosis con el $77 \%$, seguida de criptosporidiosis $(69 \%)$ brucelosis (62\%) y salmonelosis (61\%). Y los menores se obtuvieron en toxoplasmosis (33\%), rabia con el $32 \%$, teniasis y cisticercosis $(45 \%)$ y leptospirosis con el $45 \%$. Los resultados de Z demostraron diferencia entre los grupos de mayor frecuencia y los que presentaron menor frecuencia. El 31\% de los explorados aprobó el cuestionario (cuadro 3). La mayor frecuencia de aprobados fue

Cuadro 1

Entidades exploradas y resultados obtenidos por preguntas.

\begin{tabular}{llll}
\hline Temas & $\begin{array}{l}\text { Total } \\
\text { de } \\
\text { Preguntas }\end{array}$ & $\begin{array}{l}\text { Número } \\
\text { de preguntas } \\
\text { que no } \\
\text { fueron } \\
\text { aprobadas }\end{array}$ & $\%$ \\
\hline Rabia & 22 & 13 & 59 \\
Leptospirosis & 27 & 15 & 55 \\
Brucelosis & 22 & 9 & 40.9 \\
Toxoplasmosis & 13 & 11 & 84.6 \\
Teniasis y & 20 & 10 & 50 \\
Cisticercosis & & 6 & 37.5 \\
Fasciolasis & 16 & 6 & 31.5 \\
Criptosporidiosis & 19 & 5 & 45.4 \\
Salmonelosis & 11 & 75 & 50 \\
\hline Total & 150 & & \\
\hline
\end{tabular}

Fuente: encuesta

Nota: se consideró desaprobada una pregunta cuando menos del 70\% (partiendo de la muestra de 107, son 76 ó menos médicos veterinarios) la respondió correctamente. 
M Suárez-Hernández, F Llorens-Blanco, O Cepero-Rodríguez, M Retureta-Milian y col.

Cuadro 2

Aprobación del cuestionario por temas.

\begin{tabular}{lcl}
\hline Entidades & Aprobados & \% \\
\hline Leptospirosis & 48 & 45 \\
Rabia & 34 & 32 \\
Brucelosis & 66 & $62^{*}$ \\
Toxoplasmosis & 35 & 33 \\
Teniasis- Cisticercosis & 48 & 45 \\
Fasciolasis & 83 & $77^{*}$ \\
Criptosporidiosis & 74 & $69^{*}$ \\
Salmonelosis & 61 & $57^{*}$ \\
\hline
\end{tabular}

Fuente: encuesta. $\quad \mathbf{p}<0.05 *$ existen diferencias entre la aprobación de los temas de las entidades señaladas y el resto.

en el grupo que llevaba más de 15 años de graduados, no existiendo diferencia significativa.

\section{DISCUSIÓN.}

En el presente estudio se detectó que sólo el $31 \%$ de los médicos veterinarios aprobaron el cuestionario, a pesar de explorarse preguntas básicas de las principales zoonosis reportadas en la provincia. Los resultados demuestran que es necesario realizar una intervención educativa con la población de médicos veterinarios no vinculados al sector salud, con vistas a obtener mejores resultados en los programas de zoonosis en la provincia.

Se debe informar de la presente investigación a las facultades de ciencias veterinarias del país para que se realice una capacitación a los alumnos de pregrado en la salud pública veterinaria. El grupo de más de 15 años de graduado presentó la mayor proporción de aprobados, sin existir diferencia con el resto, por lo cual habrá que diseñar un estudio analítico para buscar las causas.

De acuerdo a las estrategias del Ministerio de Salud Pública de la República de Cuba, la interrelación con otros organismos del estado es necesaria para obtener resultados superiores (1).

Las principales medidas de salud adoptadas por los países miembros de la OMS, como clave para conseguir la meta de salud para todos,

\section{Cuadro 3 \\ Años de graduación y la aprobación del cuestionario.}

\begin{tabular}{lccc}
\hline Rangos & Número & Aprobados & $\%$ \\
\hline 1 a 5 años & 23 & 6 & 26 \\
6 a 10 años & 22 & 5 & 23 \\
11 a 15 años & 24 & 5 & 21 \\
Más de 15 & 38 & 17 & 45 \\
\hline Total & 107 & 33 & 31 \\
\hline
\end{tabular}

Fuente: encuesta

reconoce que la salud humana no se puede alcanzar sólo por el sector salud, pues requiere además de los sectores de desarrollo y sociales, la zootecnia y la agricultura (6).

El presente estudio se realizó con un eslabón decisivo que son los médicos veterinarios, grupo de alto riesgo de adquirir diferentes zoonosis. Además, es consultado sistemáticamente por los propietarios para recibir consejos sobre la salud animal y es vital lograr una preparación adecuada sobre la salud pública veterinaria que le permita jugar un papel importante en la prevención de las zoonosis en él y en los propietarios (7).

En diferentes países se ha demostrado la importancia que tienen los servicios veterinarios en la vigilancia, prevención y control de la rabia y leptospirosis, así como de otras zoonosis de importancia médica (8 y 9).

En las Américas se ha demostrado que para el control de la rabia la educación y promoción de la salud es necesaria. En éstas los servicios de salud animal juegan un papel decisivo ya que la rabia rural constituye un problema en diferentes países (10).

El Ministerio de Salud Publica de Holanda realizó un trabajo sobre los conocimientos de los médicos veterinarios sobre la toxoscariaris, una zoonosis de importancia médica en ese país. Si bien en nuestro estudio no se incluyó esa zoonosis, coincidimos con lo planteado por esa institución en

\section{Revista Biomédica}


Conocimientos de veterinarios sobre zoonosis.

el sentido que hay que incluir en los planes de capacitación las zoonosis para lograr resultados sostenibles en los programas de salud pública veterinaria (11).

En áreas rurales de Grecia, la educación para la salud aplicada por las autoridades de salud animal y salud pública ayudaron a reducir la fatalidad animal y disminuir los riesgos de adquirir zoonosis por las poblaciones (12).

En la literatura cubana revisada no hemos encontrado ningún trabajo donde se evalúe el nivel de conocimientos que tienen los médicos veterinarios sobre las zoonosis. En cambio, si se han realizado investigaciones con los médicos para monitorear el nivel de información sobre algunas zoonosis.

La mejor información de las zoonosis evaluadas fue la de la fasciolosis, seguida de la brucelosis. Ambas entidades han sido abordadas sistemáticamente por el Instituto de Medicina Veterinaria en programas de lucha y esto pudiera influir en un mayor conocimiento.

Es preocupante que en la rabia y leptospirosis, entidades que tienen programas conjuntos entre el Ministerio de Salud Pública y el Ministerio de la Agricultura (13 y 14) la proporción de aprobados fue bajo.

\section{CONCLUSIONES.}

1. El $31 \%$ de los estudiados aprobaron el cuestionario. El grupo de más de 15 años de graduado presentó el mayor porcentaje de aprobados, sin tener significancia estadística.

2. De las zoonosis evaluadas la mayor información fue sobre fasciolosis y brucelosis, y la menor en toxoplasmosis y rabia. Existieron diferencias significativas entre las entidades que tuvieron mayor frecuencia de aprobados y las que presentaron menor proporción.

\section{RECOMENDACIONES.}

1) Realizar una intervención educativa sobre los médicos veterinarios de otros sectores no vinculados a la salud pública.
2) Capacitar a los alumnos de pregrado en la asignatura de salud pública veterinaria.

\section{REFERENCIAS.}

1.- Ochoa R, Castanedo I, y Cobas M. Promoción de Salud. Editorial Pueblo y Educación, La Habana; 1997. p. 11-31.

2.- Van de Venter T, Prospects for the future: Emerging Problems: Chemical, Biological. Conference on International Food Trade. Melbourne, Australia, 11-15 October 1999, p. 9-11.

3.- American Committee on Arthropod-borne viruses. Identification of arbosviruses and certain rodent- borne viruses: re-evaluation of the Paradigm. Emerg Infect Dis 2001; 7:756-8.

4.- Dirección Nacional de Epidemiología. Zoonosis. Cuadro Epidemiológico de Cuba año 2000. Editorial Minsap, La Habana; 2001. p. 17-32.

5.- Dirección Provincial de Salud. Cuadro Epidemiológico de la Provincia Ciego de Ávila 2004. Editorial Trocha, Ciego de Ávila 2005. p. 1-50.

6.- Murphy F. Emerging zoonoses. Emerg Infect Dis 1998; 4:428-35.

7.- Nowotny N, Deutz A. Preventing zoonotic diseases in inmunocompromised persons. The role of physicians and veterinarians. Emerg Infect Dis 2000; 6:208.

8.- Kitala PM, McDermott JJ, Kyule MN, Gathuma JM. Community-based active surveillance for rabies in Machakos District, Kenya. Prev Vet Med 2000; 44:73-8.

9.- Sehgal S. C. Epidemiología de la leptospirosis en países en desarrollo. Reunión Científica Internacional de Leptospirosis. Ciudad de la Habana; 2001. p. 3.

10.- Thomas M, Báez M, Ruiz A. Orientaciones para la implementación y desarrollo de actividades educativas para la eliminación de la rabia humana transmitida por el perro. Organización Panamericana de la Salud, Washington; 1997. p. 7-8.

11.- Overgaauw PA, Boersema JH. Assessment of an educational campaign by practicing veterinarians in the Netherlands on human and animal Toxoscara infections. Tjidschr Biergeneeskd 1996; 121:615-8. 
M Suárez-Hernández, F Llorens-Blanco, O Cepero-Rodríguez, M Retureta-Milian y col.

12.- Hadjichristodoulou C, Soteriades E, Goutzianna G, Loukaidau M, Babalis T, Antoniou M, et al. Surveillance of brucellosis in a rural area of Greece: application of the computerised mapping programme. Eur J Epidemiol 999; 15:277-83.

13.- Cruz R, et al. Programa Nacional de Prevención y Control de la Rabia. Editorial Seruinpres, La Habana; 1998. p. 1-67.

14.- Cruz R, et al. Programa Nacional de Prevención y Control de la Leptospirosis Humana. 2da edición. Editorial Seruinpres, La Habana; 1998. p. 9-10. 